\title{
Outline Matching of the 2D Shapes Using Extracting XML Data
}

\author{
Noreddine Gherabi and Mohamed Bahaj \\ Hassan $1^{\text {st }}$ University, FSTS, \\ Department of Mathematics and Computer Science \\ \{gherabi, mohamedbahaj\} @gmail.com
}

\begin{abstract}
This paper presents an efficient shape matching method based on XML data, we extract the contour of the shape and this one is represented by set of points. Using corner detection method for representing the contour by a sequence of convex and concave segments. After, each segment is described by local and global features, this features are coded in string of symbols and stored in a XML file. Finally, using the dynamic programming, we find the optimal alignment between sequences of symbols. Results are presented and compared with existing methods using MATLAB for KIMIA-25 database and MPEG7 databases.
\end{abstract}

Keywords: XML, DOM, Shape descriptor, Shape matching, Dynamic Programming.

\section{Introduction}

Matching 2D shapes and measuring the similarity between shapes are important problems in Computer vision.

A large body of research has been devoted to shape matching, comparison and recognition. The most commonly used shape representation primitives are curves, point sets, and medial axes.

Many traditional curve matching approaches [1], [2], [3] use local invariant features (e.g., curvature) as descriptors.

Shapes have several properties that can be used for recognition and categorization, like shape, color, texture and brightness. Biederman [4] suggested that edge-based representations mediate object recognition. In his approach, color and texture of surfaces are used to define edges which are then used for recognition.

The goal of our work is to develop a descriptor based on the local and global information of the shape. These information are coded in string of symbols, these are compared using the Dynamic Programming approach. In [5, 6] dynamic programming is used to minimize a cost function that accounts for displacement of a contour in a pair of images from an image sequence. In [7] a DP approach has been used for shape matching and retrieval. The basic idea behind this approach is to represent each shape by a sequence of convex and concave segments using the inflection points extracted 
from the curvature and to allow the matching of merged sequences of small segments in a shape with larger segments in the other shape.

This paper looks into developing a shape descriptor for a contour of any shape and transforms it into string of symbols; they will be stored in an XML file. For each shape there is an XML file that corresponds to the features of the shape. Man Hing [8] uses this technique to extract the features information of the shape and represent this information in an XML format, this proposed system will use the XML (standard language) for querying different image databases.

Our approach aims to develop a simple and fast method of shape matching based on the transformation semantic data of the shape into XML format and compute the similarity between XML Files using Dynamic programming.

\section{Proposed Method}

Our approach to shape recognition is based on several steps summarized in Fig 1.
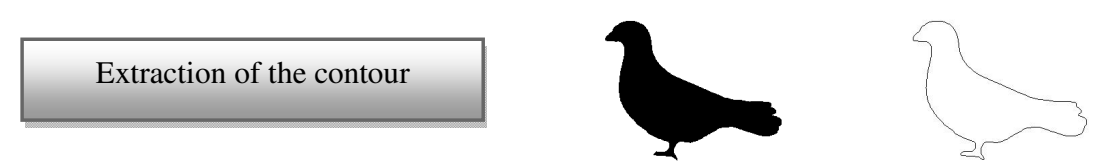

Detection of the corner and divided the contour into curves
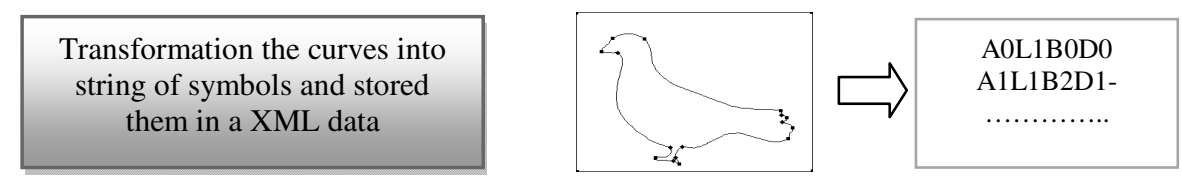

XML

DATA
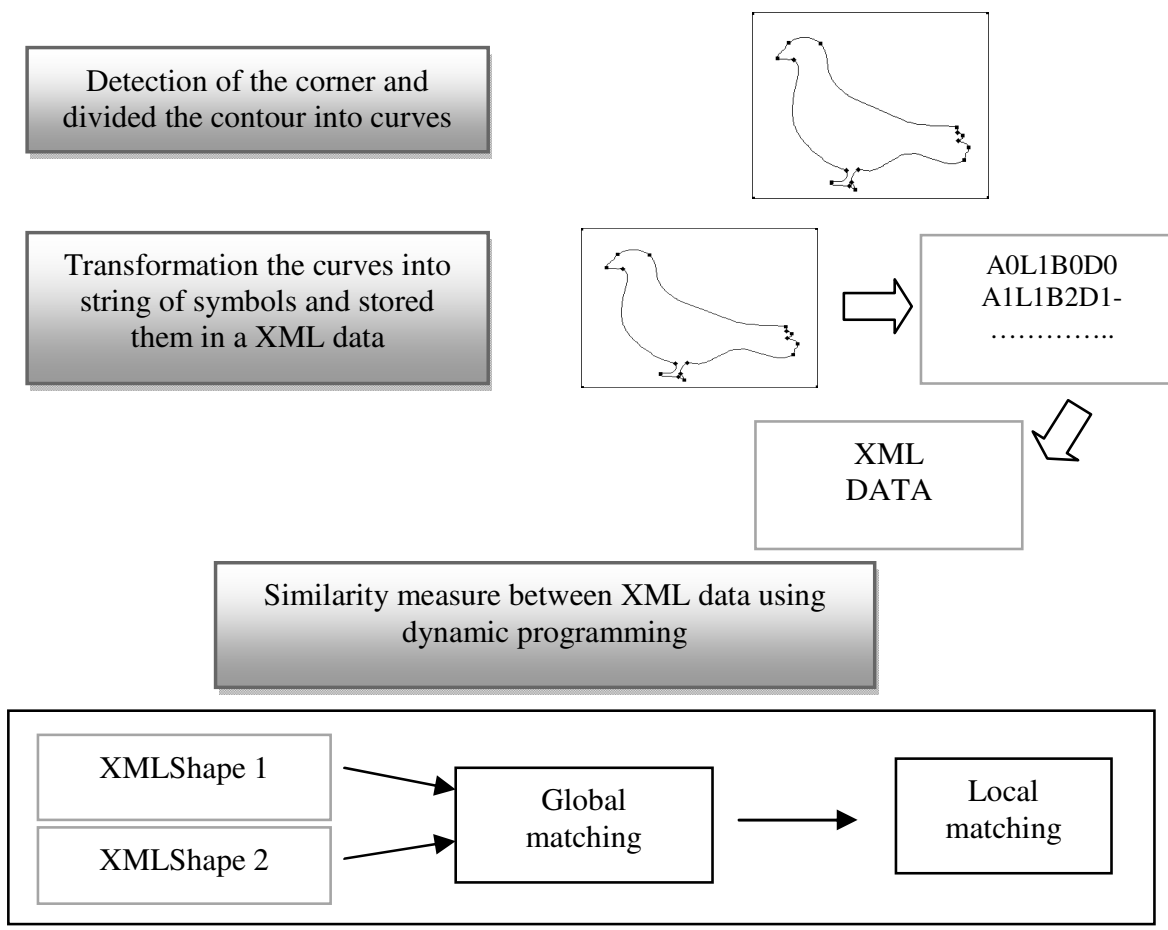

Fig. 1. A block representation of the proposed algorithm for shape matching 
The first step is to analyze the contour of the shape to be studied. The contour is retrieved and represented by sets of points. After, the contour is divided into curves by using the technique of detection corner [11], this technique is detailed in section 2.1. Then each curve is transformed into a string of symbols. The string of symbols of each curve is stored in a XML file (Section 2.2). Finally, we use the technique of dynamic programming for computing the similarity between the set of symbols stored in XML file (Section 2.3).

\subsection{Corner Detector}

Corners in images represent critical information in describing object features that are essential for pattern recognition and identification. There are many applications that rely on the successful detection of corners, including motion tracking, object recognition, and stereo matching $[9,10,11]$. As a result, a number of corner detection methods have been proposed in the past. In this paper, we use an algorithm developed by Xiao Chen and Nelson H. C. Yung [12], it works in two passes and defines a corner in a simple and intuitively appealing way, as a location where a triangle of specified size and opening angle can be inscribed in a curve. The curve has to be generated previously using an edge detector. It is not required to be a closed curve. In the first pass the sequence of points is scanned and candidate corner points are selected. In each curve point $\mathrm{p}$ the detector tries to inscribe in the curve a variable triangle $\left(\mathrm{p}^{-}, \mathrm{p}, \mathrm{p}^{+}\right)$. The triangle varies between a minimum and a maximum square distance on the curve from $\mathrm{p}^{-}$to $\mathrm{p}$, from $\mathrm{p}$ to $\mathrm{p}+$ and the angle $\alpha \leq \alpha_{\max }$ (the value of $\alpha_{\max }$ is defined) between the two lines $a$ and $b$ in Figure 2. Triangles are selected starting from point $p$ outward and the number of admissible triangles is defined. At a neighborhood of points only one of these admissible triangles is selected (See Fig.2)
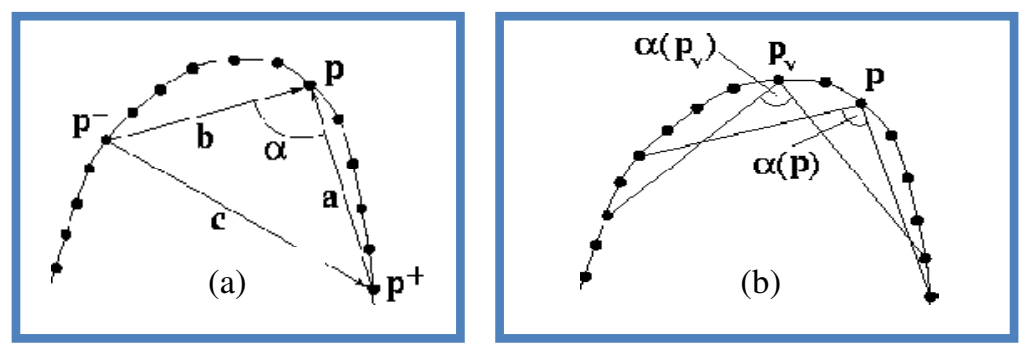

Fig. 2. Detecting high curvature points. (a) Determining if $p$ is a candidate point. (b) Testing $p$ for sharpness

\subsection{Symbolic Representation and Storing XML}

\section{Our Approach for Symbolic Representation}

The contour of the shape is retrieved and normalized to a set of points. This normalized contour used for feature extraction.

Our method uses local and global features to transform shape data into a new structure that supports measuring the similarity between shapes in an efficient manner, 
using the corner detection, the contour is segmented into a set of primitives (line, convex and concave curves) and described by the features: $A_{i}, 1_{i}, \operatorname{Dg}_{i}, \beta_{i}$, where :

$A_{i}$ is the area of the triangle enclosed the chord and the arc between the inflection points $\mathrm{Pi}$ and $\mathrm{Pi}+1$, this area is calculated using Heron's formula (See Fig 2):

$$
\text { A rea }=\sqrt{s(s-a)(s-b)(s-c)}
$$

Where: $\quad s=\frac{a+b+c}{2}$ is the Semiperimeter, or half of the triangle's perimeter.

$l_{i}$ is the length of Curve $\left(C_{i}\right)$. Dgi is the Degree of concavity or convexity $\left(\mathrm{Dg}_{\mathrm{i}}=\mathrm{d}_{\mathrm{i}} \mathrm{l}_{\mathrm{i}}\right)$ is computed as the ratio of the maximum of distances from points on the curve to associated chord and the distance of the chord of $\left(\mathrm{C}_{\mathrm{i}}\right)$.

$\beta \mathrm{i}$ is the angle traversed by the tangent to the segment from inflection point $\mathrm{Pi}$ to inflection point $\mathrm{Pi}+1$ and shows how strongly a section is curved( See Fig. 3).

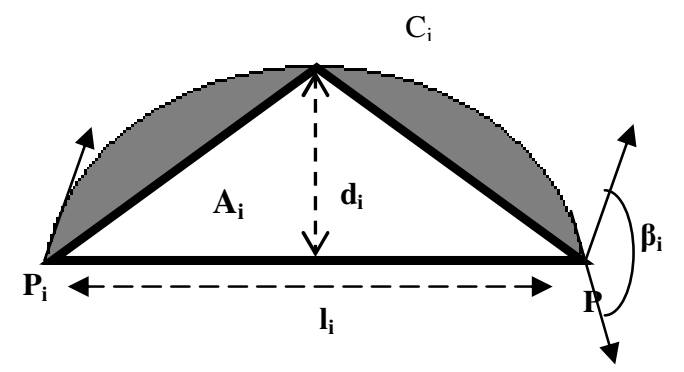

Fig. 3. shape descriptor of the curve Ci

Now we will see how to transform the shape into a set of symbols.

The area $\mathrm{Ai}$ is computed and quantized in three bins (A0, A1, A2) corresponding to a zero, small and large area. The same, for each curve of the contour, the length of the segment $[\mathrm{Pi}, \mathrm{Pi}+1]$ is computed, this normalized distance li is quantized in three bins (L1, L2, L3) corresponding to a small, medium and large distance of li. Next, the angle $\beta \mathrm{i}$ is computed and quantized in different five bins between $[0, \pi](\mathrm{B} 0, \mathrm{~B} 1, \mathrm{~B} 2$, $\mathrm{B} 3, \mathrm{~B} 4), \mathrm{B} 0$ for $\beta \mathrm{i}=0$. Finally, the values Dgi of each curve is computed and quantized in three bins (D0, D1, D2) corresponding to zero, a small and large values of Dgi.

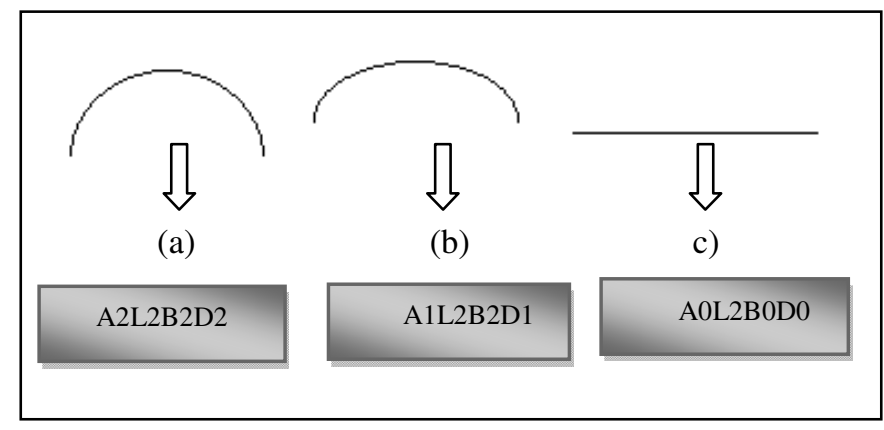

Fig. 4. The mapping obtained by the algorithm of a given contour into a string of symbols 
Our algorithm converts the contour of the shape into sequences of symbols, for example the Mapping obtained by the algorithm of a curve (a) illustrated in figure 4 is : A2L2B2D2, A2 corresponding to a large area, L2 corresponding to a medium distance of $\mathrm{Li}, \quad \beta \mathrm{i}$ is quantized in the bin B2 and D2 corresponding to a large value of Dgi.

\section{Writing in XML Data}

In this paper we propose XML language for describing the features of the shape structured in order where each contour is associated one descriptor written in a specific file XML. This technique for converting into XML is already used in our previous work [12].

To write the shape descriptor, each curve is represented by a set of parameters, these parameters are encoded using XML tags. to construct the XML structure we used the technique of DOM in Matlab. We use the syntax of XML to write our outline of the shape as follows:

The curve is defined by its type and described by the parameters $\left(\mathrm{A}_{\mathrm{i}}, \mathrm{L}_{\mathrm{i}}, \mathrm{Dg}_{\mathrm{i}}, \beta_{\mathrm{i}}\right)$

- Concave/ Convex/Right curve

$<$ C number $=$ ', $>/ /$ curve and its number in the outline

$<$ Type $>\mathrm{CC}$ or $\mathrm{CV}$ or $\mathrm{R}<\mathrm{Type} />/ /$ Type of curve (concave/convex) or right line

$$
\begin{array}{rlrl} 
& <\mathrm{Ai}> & </ \mathrm{Ai}>\text { area } \mathrm{Ai} \\
<\text { li }> & </ \text { li }>\text { length of curve } \\
<\text { beta_i } & </ \text { beta_i }>\text { angle } \beta_{\mathrm{i}} \\
</ \mathrm{C}> & <\text { Dgi }> & <\text { Dgi }>\text { degree of gravity }
\end{array}
$$

An iterative process is presented to describe the shape using XML, this process is in the following algorithm:

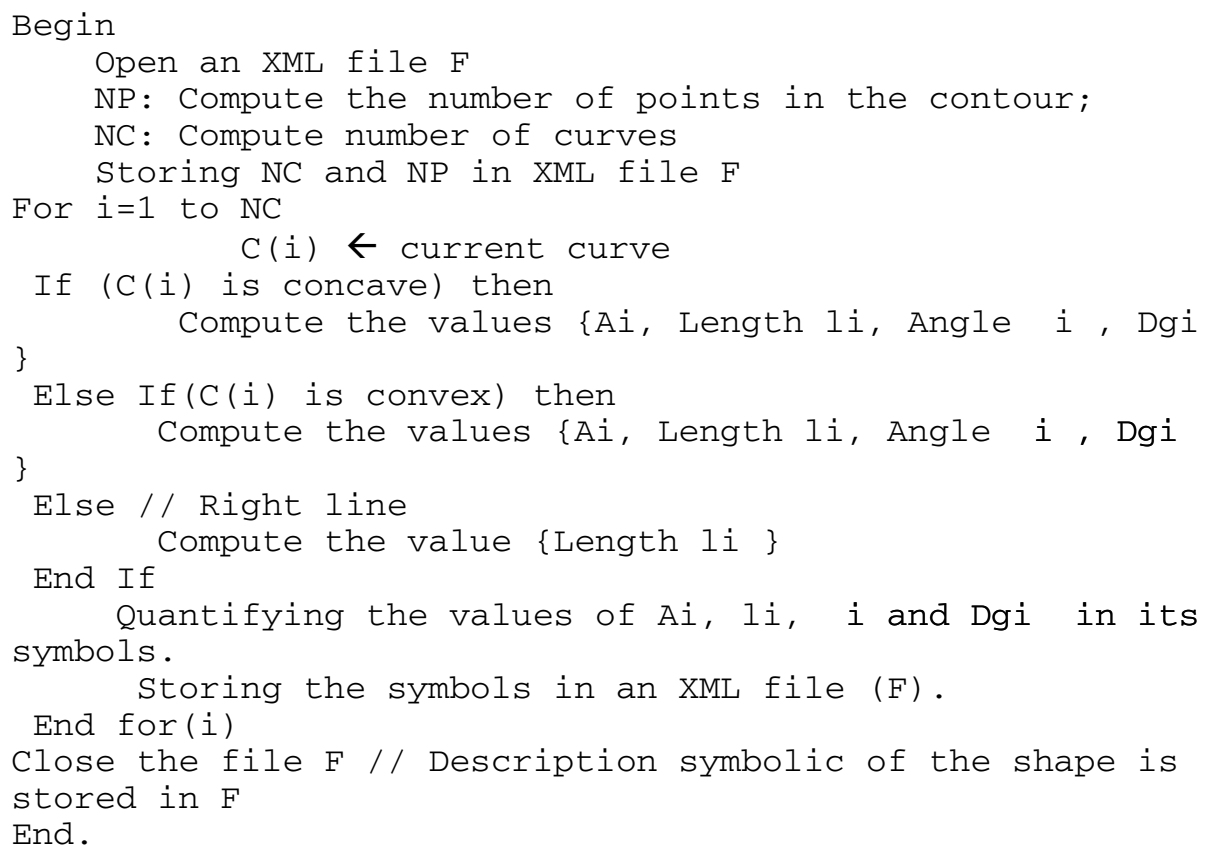


Our algorithm creates an XML file for each shape, for example the XML descriptor computed for curve (a) illustrated in figure 4 is:

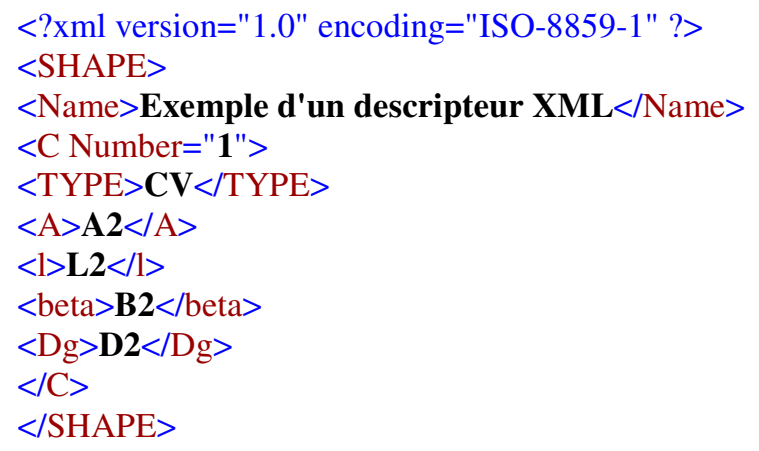

\subsection{Matching Shape}

\section{Global Matching}

At this level, we are interested only in global information's which characterize the general aspect of an object. At first, matching is done with comparing the number of different components of the outline shape descriptor.

From the XML descriptor it is easy to extract the following indices:

- Number of inflection points in the contour and Number of curves: Computed as the number of tag $<\mathrm{C}>$.

- The order of each Curve defined by its number.

- Number of convex/ concave curves: computed as the number of type CV/ CC.

- Number of right lines: computed as the number of type R.

In some cases both of the contours have the same global descriptor; in this case the global matching is not possible. At the issue of global matching using an XML descriptor is the obtaining of a list of candidate couples of contours that constitute the input for the next step.

Using an XML descriptor reduces the execution time and research to a large database

\section{Local Matching}

After the outlines stored in XML files, their similarity can be evaluated by an appropriate comparison of each string of symbols stored in the tags $<\mathrm{C}\rangle$.

All string of symbols stored in the tags $<\mathrm{C}>$ be extracted from the XML file of the first shape and then compare them with other strings of the other XML file of the second shape.

We use the technique of dynamic programming for a good matching between strings of symbols. The dynamic programming can find the best alignment between two strings with different lengths. When sequences of strings are aligned, sequence 
alignment scores are computed. The system can find similar sequences by sorting the alignment score. In this paper, we use the algorithm of Levenshtein Edit distance [14], this technique was modified by adding cost of similarity between the symbols. The edit distance between two strings is given by the minimum number of operations needed to transform one string into the other, where an operation is either an insertion, deletion, or a substitution of a single character.

We construct a matrix $\mathrm{D}[0,--, \mathrm{m} ; 0,--, \mathrm{n}]$. The matrix $\mathrm{D}$ is computed using the recurrent equation:

$$
\mathrm{D}(\mathrm{i} ; \mathrm{j})=\min \left\{\begin{array}{l}
\mathrm{D}(\mathrm{i}-1 ; \mathrm{j}-1)+\mathrm{F} ; / / \text { a substitution } \\
\mathrm{D}(\mathrm{i}-1 ; \mathrm{j})+\mathrm{w} ; / / \text { a deletion } \\
\mathrm{D}(\mathrm{i} ; \mathrm{j}-1)+\mathrm{w} ; / / \text { an insertion }
\end{array}\right\}
$$

$\mathrm{D}(\mathrm{i}, \mathrm{j})$ represents the score for the matrix position, $\mathrm{W}$ represents a gap of penalty score its value equal to "2" and $\mathrm{F}$ represents the match/mismatch score.

This is a dynamic programming algorithm in Matlab language:

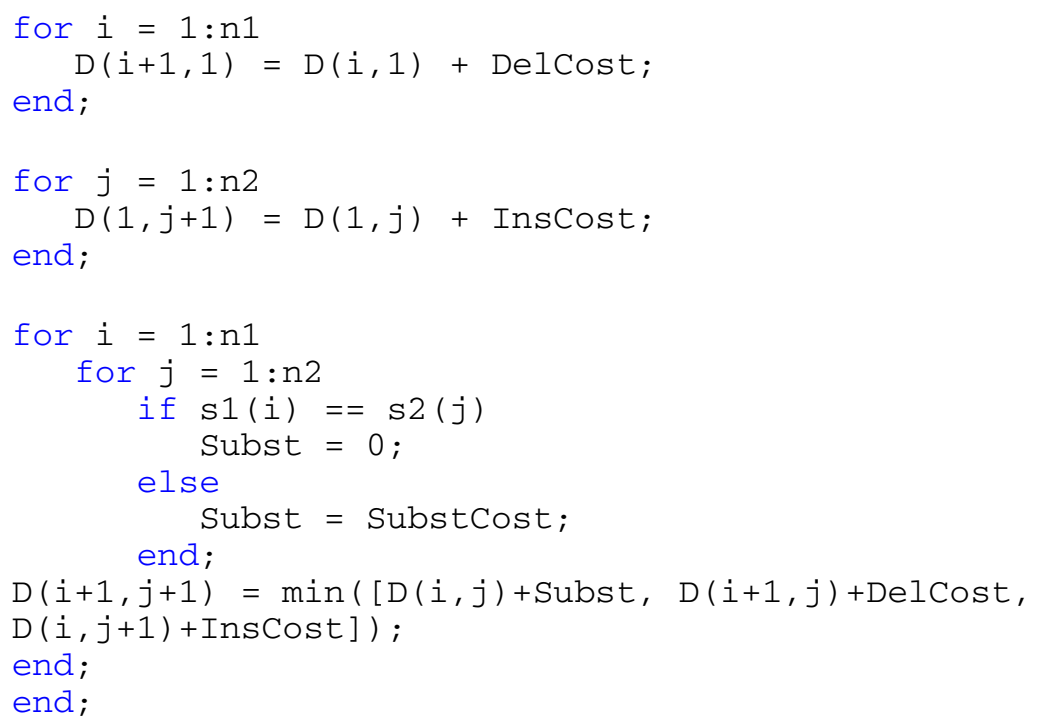

The first step for DP algorithm is to create a matrix with $\mathrm{M}+1$ columns and $\mathrm{N}+1$ rows where $\mathrm{M}$ and $\mathrm{N}$ correspond to the size of the sequences to be compared. This DP algorithm has been modified to take into account the differences resulting from the quantification of areas, distances and angles. A smaller weight or penalty (with a value lower than one) for the substitution of two adjacent symbols was introduced; for example the distance between $\mathrm{A} 1$ and $\mathrm{A} 2$ was taken to be equal to 0.5 and $\mathrm{A} 1$ and $\mathrm{A} 3$ equal to 1, and similarly the distance between B1 and B2 or L1 and L2 or D1 and D2 was taken to be equal to 0.5 . The compute starting in the upper left hand corner in the matrix and finding the minimal score for each position in the matrix. The minimal score is calculated using the formula (2). Therefore, the algorithm helps the system avoid computing an exponentially large number of comparisons. When sequences of 
strings are aligned, sequence alignment scores are computed. String sequences are matched well for lower alignment scores. The system can find sequences that are similar to a query key sequence and the minimum score is selected.

Consider the sequence of the shape (a) presented in figure 4 is a query key sequence and comparing him with sequences of the two shapes (b) and (c) respectively:

The score matrix for two cases is as follows:

Contour (a) with (b)
\begin{tabular}{|c|c|c|c|c|}
\hline & A2 & L2 & B2 & D2 \\
\hline A1 & 0.5 & 2 & 2 & 2 \\
\hline L2 & 2 & 0.5 & 2.5 & 4 \\
\hline B2 & 2 & 2.5 & 0.5 & 2.5 \\
\hline D1 & 2 & 4 & 2.5 & 1 \\
\hline
\end{tabular}

Contour (a) with (c)
\begin{tabular}{|c|c|c|c|c|}
\hline & A0 & L2 & B0 & D0 \\
\hline A1 & 0.5 & 2 & 2 & 2 \\
\hline L2 & 2 & 0.5 & 2.5 & 4 \\
\hline B2 & 2 & 2.5 & 1.5 & 3.5 \\
\hline D1 & 2 & 4 & 3.5 & 2 \\
\end{tabular}

Fig. 5. An example showing how to compute the edit distance between two strings. The last cell shows the distance computed for these two strings.

After filling the score matrix, the minimum alignment score for the sequence (a) with sequence (b) is 1 and the minimum alignment score for the sequence (a) with sequence (c) is 2, so the shape (a) is more matched with shape (b) .

\section{Experiments}

The method has been tested on a set of MPEG-7 and KIMIA-25 shapes illustrated by Fig.6. For each shape, contours of objects have been extracted. After that, we determinate for each contour, the inflection points using corner detection. In Fig.7 we illustrate an example of dividing the contour of the shape into set of primitives (convex or concave curves or lines)

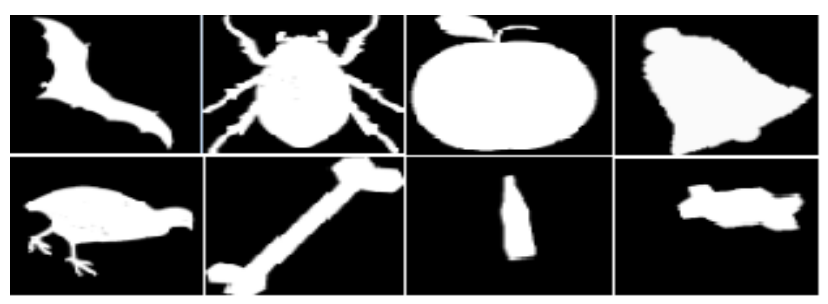

(a)
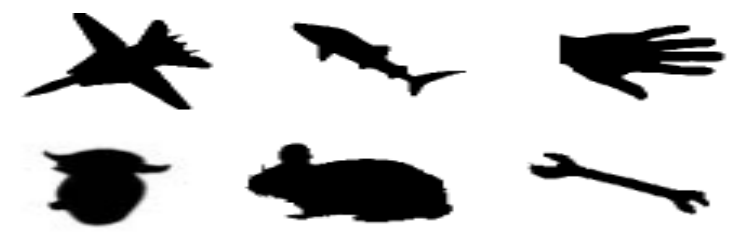

(b)

Fig. 6. Some of the objects in the (a) MPEG-7 database and (b) KIMIA-25 database 


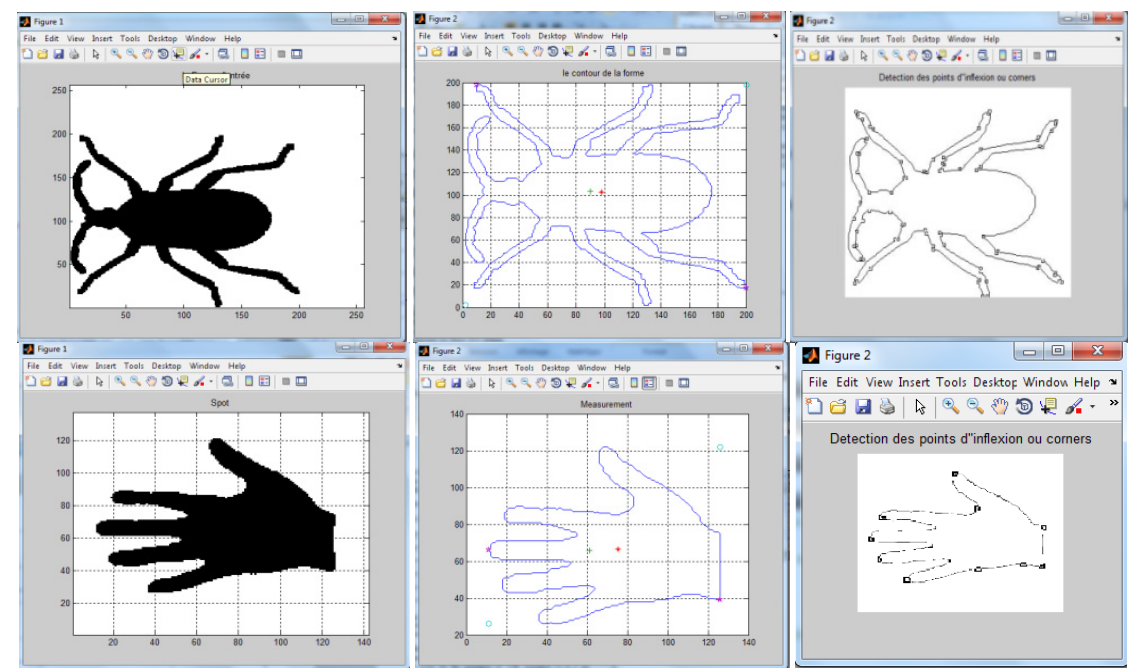

Fig. 7. Extraction the contour from two shapes in databases MPEG-7 and KIMIA-25 and detection the inflection points

The system creates an XML file for each shape and each shape is indexed by its $\mathrm{xml}$ file.

We took the XML file for some shapes and were used as reference XML files for experimentation; the number of reference XML files is defined as $\mathrm{K}$. The percentage of matches between the reference XML and other files is obtained, and we computed the percentage of matching for different databases and different values of $\mathrm{K}$.

The number of iteration using in this paper is 10 and the value of the parameter $\mathrm{K}$ is taking equal $(3,7,15,20,50)$.

Results are presented as a percentage. The graph in figure 8 shows the score of matching for different values of $\mathrm{K}$ in two databases MPEG-7 and KIMIA-25.

The best match is achieved for $\mathrm{k}=20$ (98.7\% for KIMIA-25) and (98.9\% for MPEG-7). These results are compared with some old methods and techniques in MPEG-7.

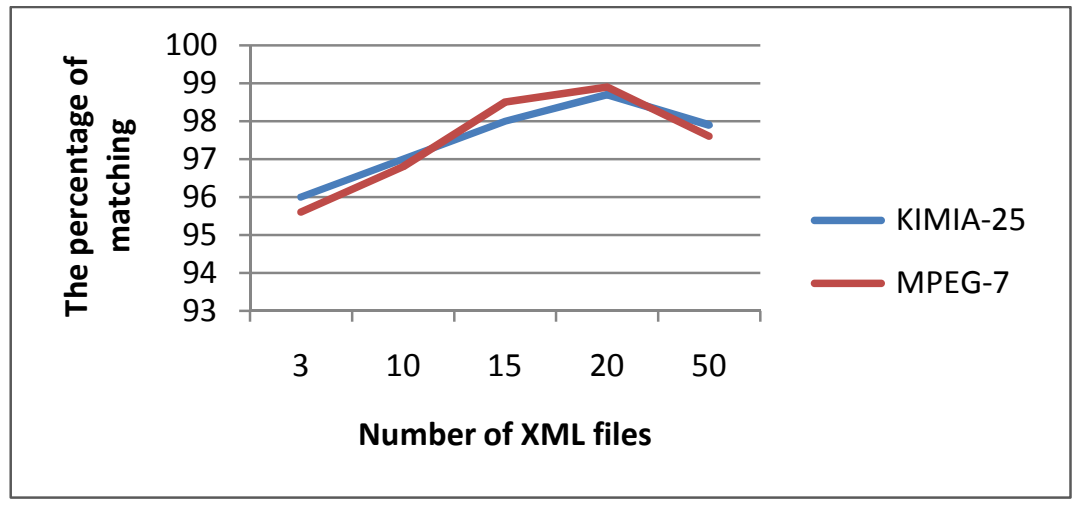

Fig. 8. Percentage of matching for five values of K in MPEG-7 and KIMIA-25 


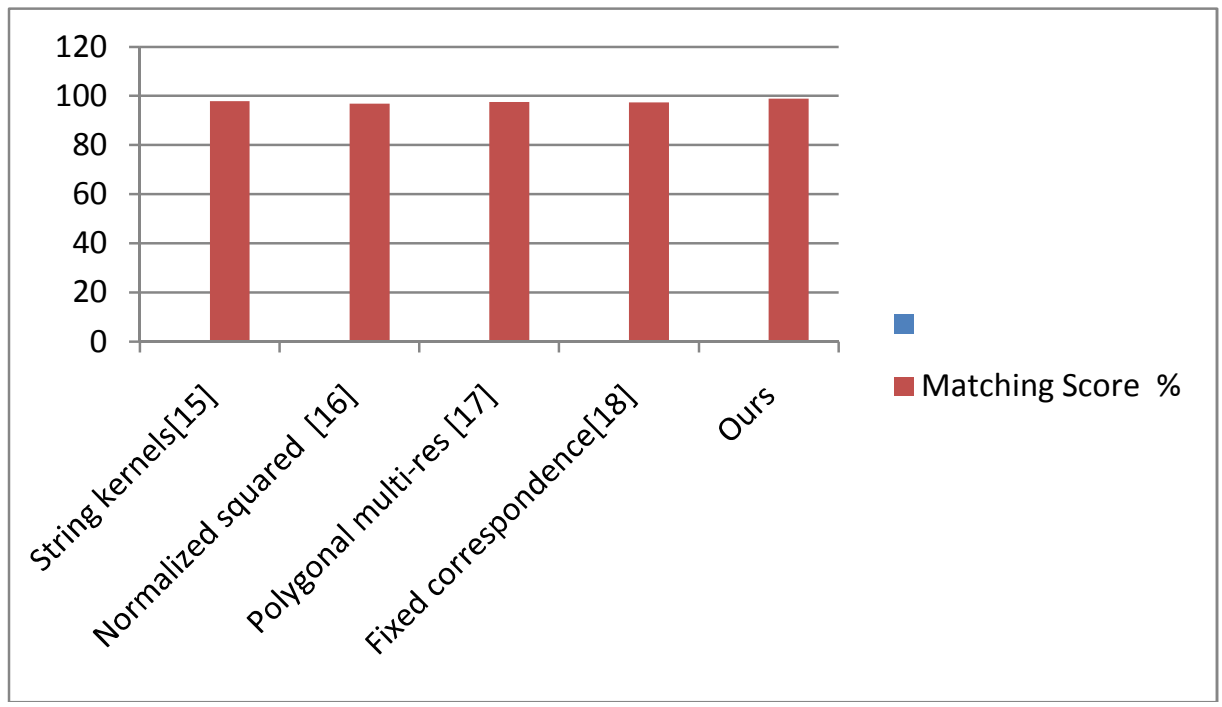

Fig. 9. Comparison of results in MPEG-7database

Our algorithm was compared with some old methods and our result is advanced with a little percentage compared to other solutions (Fig.9).

\section{Conclusion}

We have presented a new technique for shape matching. A key characteristic of our approach is the transformation of the shape features in XML file and then compare these XML files using dynamic programming. After different kinds of experimentation on MPEG -7 and KIMIA-25 Shape database, the proposed method has given interesting results over the existing methods.

\section{References}

1. Wolfson, H.J.: On Curve Matching. IEEE Trans. on Pattern Analysis and Machine Intelligence 12, 483-489 (1990)

2. Kishon, E., Hastie, T., Wolfson, H.J.: 3-D Curve Matching using Splines. J. of Robotic Systems 8, 723-743 (1991)

3. Barequet, G., Sharir, M.: Partial Surface Matching by Using Directed Footprints. In: Symposium on Computational Geometry, pp. C-9-C-10 (1996)

4. Biederman, I., Ju, G.: Surface versus edge-based determinants of visual recognitions. Cognit. Psychol. 20, 38-64 (1988)

5. Geiger, D., Gupta, A., Costa, L.A., Vlontzos, J.: Dynamic Programming for Detecting, Tracking amd Matching Deformable contours. IEEE Trans. on Pattern Analysis and Machine Intelligence 17(3), 294-302 (1995) 
6. Floreby, L.: A Multiscale Algorithm for Closed Contour Matching in Image Sequence. In: IEEE Intern. Conf. on Pattern Recognition, pp. 884-888 (1996)

7. Petrakis, E.G.M., Diplaros, A., Milios, E.: Matching and retrieval of distorted and occluded shapes using dynamic programming. IEEE Trans. Pattern Anal. Mach. Intell. 24(11), 1501-1516 (2002)

8. Yu, M.H., Lim, C.C., Jin, J.S.: Shape similarity using XML and portal technology. In: Visual Information Processing, VIP, Sydney, Australia (2006)

9. Fung, G.S.K., Yung, N.H.C., Pang, G.K.H.: Vehicle shape approximation from motion for visual traffic surveillance. In: Proc. IEEE 4th Int. Conf. on Intelligent Transportation Systems, pp. 201-206 (2001)

10. Manku, G.S., Jain, P., Aggarwal, A., Kumar, A., Banerjee, L.: Object tracking using affine structure for point correspondence. In: Proc. 1997 IEEE Comput. Soc. Conf. on Computer Vision and Pattern Recognition, pp. 704-709 (1997)

11. Serra, B., Berthod, M.: 3-D model localization using highresolution reconstruction of monocular image sequences. IEEE Trans. Image Process. 6(1), 175-188 (1997)

12. He, X.C., Yung, N.H.C.: Corner detector based on global and local curvature properties. Optical Engineering 47(5), 057008-1-057008-12 (2008)

13. Gherabi, N., Bahaj, M.: A new shape descriptor using XML. IJCSE 3, 1369-1376 (2011)

14. Ristad, E.S., Yianilos, P.N.: Learning string edit distance. IEEE Trans. Pattern Anal. Mach. Intell. 20(5), 522-532 (1998)

15. Daliri, M.R., Delponte, E., Verri, A., Torre, V.: Shape Categorization Using String Kernels. In: Yeung, D.-Y., Kwok, J.T., Fred, A., Roli, F., de Ridder, D. (eds.) SSPR 2006 and SPR 2006. LNCS, vol. 4109, pp. 297-305. Springer, Heidelberg (2006)

16. Super, B.J.: Learning chance probability functions for shape retrieval or classification. In: Proceedings of the IEEE Workshop on Learning in Computer Vision and Pattern Recognition (June 2004)

17. Attalla, E., Siy, P.: Robust shape similarity retrieval based on contour segmentation polygonal multiresolution and elastic matching. Pattern Recognition 38(12) (2005)

18. Super, B.J.: Retrieval from shape databases using chance probability functions and fixed correspondence. Int. J. Pattern Recognition Artif. Intell. 20(8), 1117-1137 (2006) 